\title{
ICHTHARGAN. ITS USE IN NOSE AND THROAT DISEASES.*
}

BY BEAMAN DOUGLAS, M.D., NEW YORK CITY.

Professor of Diseases of Nose and Throat, N. Y. Post Graduate Medical Bchool and Hospltal

Ichthargan was sent me by the Ichthyol Company of Hamburg, for the purpose of testing its value in nose and throat cliseases. For the past year this compound of our two valued drugs, silver and ichthyol, has been used in the clinics where I work and its effects have been carefully watched by assistants. The observations compiled by me are the result of the work of my two assistants, Dr. J. W. MeCready and Dr. Colin Begg, to whom I give full credit for their work with this remedy. The principal contributions to literature concerning this drug are by Dr. Aufrecht, consisting of reports of experiments on the lower animals; clinical work has also been done by Drs. Leistekow and H. Loenstein. So far as I am informed there is no rhinological literature on the subject.

Ichthargan is a compound of silver and ichthyol. Its chemical name is silver-thio-hydrocarbo-sulphonate and it is a brown, amor. phous powder, odorless and stable. It is casily soluble in water, glycerin, and diluted alcohol; but in insoluble in absolute alcolool; ether and chloroform. The aqueous solution becomes gradually darker when exposed to light; but if kept in amber-colored bottles it undergoes no change. A concentrated solution is precipitated by a solution of sodium chloride, and also by a solution of albumen; but the latter precipitate is redissolved by an excess (of albumen). Ichthargan contains 30 per cent of silver and 15 per cent of sulphur, both in organic combination with the bases from the ichthyol sulphonic acid. It is thus seen that in its silver contents ichthargan is the strongest of all the silver compounds; argonin containing 4.2 per cent of silver; argentamin, 6.3 per cent ; protargol, 8.3 per cent, and largin, I I I per cent.

It was to be expected a priori that this compound would prove a strong bactericide, but it still had to be ascertained whether it possessed the strong antiphlogistic, secretion-diminishing, and penetrating properties that are so eminently peculiar to ichthyol. A scries of experiments were undertaken by Prof. Aufrecht (Deut. md. Woch. No. I3, 1900), with a view of throwing some light on

"Read before Section of I,aryngology, N. I. Academy of Medicine, March 24, 1902. 
the above points. To ascertain the penetrating properties of icllthargan picces of rabbit liver were immersed in a 0.5 per cent ichthargan and the same of silver nitrate respectively. After sixteen hours they were removed and treated with ammonium sulphicle. The pieces from the ichthargan solution contained a brown. ish black percipitate throughout their entire mass, while those from the silver nitrate had a black coloration of silver sulphicle on their surface only. This shows the greater penetrating power of ichthargan over silver nitrate. Further experiments show that it had in $0.3-0.5$ per cent solutions, it had the power to prevent decomposition in meat, boutlion, urine and other organic liquids. In ntmerous experiments with anthrax bacilli, gonococci, streptococci, staphylococci, typhoid and diphtheria bacilli, it was shown to have a much higher bactericidal power than silver nitrate. It was demonstrated as less toxic than the silver in experiments on frogs, guinea pigs, rabbits and dogs."

The toxic close according to Dr. Aufrecht, is between . IO-. I5 centigrams for every kilo. of weight, while that of silver is .015, actol .035, irtol .02, protargol .04. This shows that the other silver salts are much more toxic. The investigator himself took .3 of ichthargan on three successive days and on the fourth day, .5 without unpleasant effects. He concludes that the drug when administered by mouth or subcutaneously, is not followed by dangerous results. In intravenous injection the ichthargan is more toxic, but is less so than the silver preparations.

Ichthargan for nose and throat work must be used in preparations from I-50 to I-IO. The strength of I-20 has been found best for general use. Water or glycerine or a mixture of both make a convenient vehicle for the drug in practice. In the clinic work both glyccrine and watery solutions were used so that the exact effect of the drug in both solutions could be determined, one being substituted for the other as soon as a particular effect was noticed in order to determine whether any of the effect observed was due to the glycerine menstruum. This is necessary in many cases as glycerine is an active remedial agent. It may be mentioned here that it was found best to keep the bottles containing the drug in a dark place, to prevent precipitation along the sides of the bottles of what looked to be metallic silver. When the drug is applied as a powder it causes smarting, burning and sneezing and should never be used in this form. It is not strong enough to be a cauterant and yet is strong enough to produce considerable irritation. 
Effects. Watery solutions of . I to .3 per cent seem to liave no other effect than to increase the flow of mucus from the nose. There was very little irritation and only a moderate amount of smarting for about a minute. The same percentage dissolved in glycerine showed only the effects of the glycerine. A I per cent solution was more irritating, but no vascular clanges were observed in the nasal mucous membrane and the secretion was but little more than that produced by the lower per cents. An interesting point in this connection is that some patients.complained of the use of these solutions, while others did not complain at all. In ro per cent solutions the patients complained of disagreeable taste and nasal irritation lasting over 15 minutes, sometimes as long as two hours. This strength solution caused some congestion of the membrane a few minutes after its application, with profuse nasal clischarge and lachrymation with some sneezing. After considerable experimenting it was found that the most generally satisfactory solution was 4 per cent. This produced general!y a slight burning and smarting, never enough to amount to pain, even in acute coryza cases, where the nose was hypersensitive. There was no over-reaction from the 4 per cent solution. After application of the solution to a congested area there was noticed an anemia with some contraction and shrinkage of the mucous membrane. There seemed to be some slight anesthesia produced at the same time. We never noticed any interference with the nutrition of the tissues. The anemia produced was not particularly marked in all cases, while in others it was almost as pronounced as that produced by suprarenal. In still other cases there was no vascular clange observed. Almost all drugs used in the nose produced either hyperemia or anemia of the membrane and this effect can not be claimed as one of the special effects of ichthargan. Occasionally simple irritation of the nasal mucous membrane will cause anemia of the part and probably the slight astringent action of this drug, together with the mechanical effect of its application causes the anemia noticed. The anesthesia noticed cannot be put to any practical use since it was so slight and was possibly only the loss of sensitiveness often found in anemic tissue. Its action, however, was very prompt, the effect being produced in 20 seconds and lasting from 15 to 20 minutes. This is especially true of the contracting power of the drug and its control over hyperemic nasal vessels. Not having used it in the normal nose, I can only speak of its use in pathological conditions. 
It seems to be useful for the effect which has (I) an anesthetic, (2) antiseptic, (3) antipllogistic, (4) stimulant, (5) alterant and (6) as a modifier of nasal secretion. The anesthetic effect is not marked, and is secondary to its effect on the blood vessels. Its antiseptic effects are not only seen in culture tubes, but in experiments in the nose in cases of atrophic rhinitis, the odor is materially lessened in a short time. As an antiphlogistic it has an important action on the respiratory mucous membrane. Its first effect is to prorluce anemia. This is followed, under strong solutions, by no reaction, the parts simply returning to their normal condition. When stronger solutions are used the irritant effect is more marked, producing congestion in from half an hour to an hour after application. This excessive congestion disappears in I5 minutes and the circulation in the nose seems to be improved afterwarcls. When ichthargan has been used for some time a tolerance is established. This is followed by improved blood vascular tone and clearing up of the congestion.

These effects have been observed not only in acute congestive conditions, but also in chronic congestion accompanying the active hyperemic stages of inflammation.

As a stimulant the drug acts in two ways. First as an alterant to produce more healthy circulation, diminishing congestion, lessening exudation and it also acts probably directly on the cell protoplasm itself. In modifying secretion it seems to act by lessening leucocytosis. Under its use muco-purulent secretions become mucoid and are soon normal.

Comparison with silver. The astringent and antiphlogistic effects of this drug are about the same as the silver salts. Compared with silver nitrate it may be said to be I-Io as irritating, but in strong solutions or as a powder it never cauterizes. This must not be forgotten. The question as to whether the ichthyol effects can be observed is of slight interest. One can state that the alterant effects and the tendency to modify secretion is not as much clue to the silver as to the ichthyol. However, if we acknowledge that the stimulant alterant and antiphylogistic effects represent the ichthyol, we still find the advantage with the ichthargan solution in that it is odorless. The preparation cannot be said to have an ichthyol taste, the petroleum flavor being modified by the metallic taste of the silver. It is clear that this remedy is not as useful as silver when cauterization is necessary, but it has the advantage in that it is non-irritant and non-cauterizant in fairly strong solutions. 
If we add the good effects of ichthyol to the desirable effects of silver we have a preparation which can be used to great advantage in some cases. It will be found most useful in acute catarrhal rhinitis in a 4 per cent solution; in cases of hypertrophic rhinitis which are not particularly hyperplastic, and where obstruction results from disturbed circulation; here its antiphlogistic and alterant properties are valuable. In atrophic rhinitis cases if this remédy were used in weak solutions as an irrigation, I : 5,000, and afterwards in 4 to Io per cent solutions in water or glycerin, it would seem par excellence, the remedy most inclicated for atrophic mem. brane. In this disease we require some antiseptic, something to correct the circulation, a stimulant for the functional parts of the mucous membrane, and an alterative effect on the connective tissue. The leucocytic infiltration which is a prominent feature of atrophic cases is also benefited by ichthargan. It would seem to be clearly indicated in acute and cluronic conditions of the naso-pharynx as well as inflammation of the pharyngeal bursa. It conld not be used in the accessory sinuses except, perhaps, after operations, when it could be used in ro per cent solution on cotton pledgets applied to the granulations which often appear. In tonsillitis and inflammations of the lingual tonsil, better results may be expected from the other silver salts. Ichthargan can safely be recommended in certain diseases of the larynx, in acute catarrhal laryngitis in adults in 4 to 8 per cent solutions sprayed over the parts, in laryngitis sicca in a ro per cent glycerin solution, and in chronic atrophic tracheitis where there is abundance of dry saliva and scales.

In our experience ichthargan has acted well in acute rhinitis in a 2 per cent solution and in an ointment with vaseline. In hypertroplic cases the best results were from the use of a 4 per cent solution, the secretion in these cases being lessened, the purtulent matter of the muco-purulent secretion disappearing leaving the secretion diminished in quantity and more mucoid in character. In cases of granulation tissue formation after operation a 4 , IO or 20 per cent glycerin solution has been of the greatest value in healing the exuberant granulations and modifying the secretion. Some of the most brilliant results have been obtained in these cases. In atrophic cases. it seems to attain its most effective results. It acts like a charm since it combines the effects of the best two remedies. It may be said that in atrophic cases to arrest suppuration, remove scabs and stimulate the membrane to a healthy action, it is unequalled. We have treated at least fifty cases of this disease and 
are in a position to state that the results with ichthargan have been more satisfactory than with any other form of treatment. The two following cases are taken at random to illustrate the effects of this drug.

Case I. Mrs. S. H., 24, for several years has been treated for atrophic rhinitis without deriving any marked benefit. The patient complains of dryness, itching, scabbing, constant desire to blow the nose, and bleeding if scabs are detached. On examination the nasal mucous membrane was dry and covered with scabs. There is atrophy of the turbinates on both sides, the region being covered with scabs and superficial ulcers. There are dry, hard scabs along the septum, leaving bleeding areas on removal. The nose was thoroughly cleaned and an application of a 4 per cent solution of ichthargan was used four times a week. The patient was given a 2 per cent ointment of ichthargan in vaseline to be introduced into the nose twice a day. The nose was not to be washed out at home. At the end of three weeks the distressing symptoms had disappeared, the scabs were gone and mucous membrane appeared healthy for an atrophic case. On examination two months later there were still no scabs, although the only treatment carried out had been the occasional application of the ointment at night.

Case II. Mrs. N.; 45, an old, neglected case of atrophic rhinitis. The patient complained of dryness, itching and disagreeable odor. Scabs were frequently discharged on blowing the nose. On examination the mucous membrane was found very dry and parchmentlike with marked atrophy of the turbinates and atrophic nasopharyngitis and pharyngitis. There were small ulcers under the scabs along the septum and hard, dry scabs with ulcers on other parts. The treatment consisted in thorough cleansing of the nose and naso-pharynx, with a solution of boric acid. The crusts were removed with a cotton applicator. The ulcerated spots were touched with a Io per cent watery solution of ichthargan, and the entire nose and pharynx sprayed with a 4 per cent solution. This was done four times a week for five weeks, at the end of which time the mucous membrane was more vascular, and the ulcers and scabs had disappeared. One or two small scabs remained along the septum. After this treatment the patient only required the tuse of a 2 per cent ointment daily. The patient was seen one month later and showed decided improvement. There was no odor, no scabs nor ulcers, and the patient was discharged with instructions to use the ointment once daily.

We have also used this remedy in watery and glycerine solutions in various forms of nasal ulceration, in apthous disease, and syphilitic ulcers of the pharynx and mucous patches of the pharynx and mouth, in acute as well as syphilitic and tubercular laryngitis, and it has been as satisfactory as any silver preparation we have ever used. It would seem that this remedy were worthy of further trial in nose and throat diseases, and it is with confidence that we recommend it to all members of this Section. 